Voix et Images

voixetimages

\title{
Le coeur battant du poème
}

\section{Michel Lemaire}

Volume 19, numéro 3 (57), printemps 1994

Science et fiction au Québec : L'émergence d'un savoir

URI : https://id.erudit.org/iderudit/201124ar

DOI : https://doi.org/10.7202/201124ar

Aller au sommaire du numéro

Éditeur(s)

Université du Québec à Montréal

\section{ISSN}

0318-9201 (imprimé)

1705-933X (numérique)

Découvrir la revue

Citer cet article

Lemaire, M. (1994). Le coeur battant du poème. Voix et Images, 19(3), 641-644. https://doi.org/10.7202/201124ar d'utilisation que vous pouvez consulter en ligne.

https://apropos.erudit.org/fr/usagers/politique-dutilisation/ 


\section{Le cœur battant du poème}

\section{Michel Lemaire, Université d'Ottawa}

L'étude de la versification est sans doute l'un des domaines de la recherche littéraire les moins à la mode aujourd'hui. On imagine un vieux monsieur à barbiche qui compte ses pieds sur ses doigts et compulse des traités poussiéreux. Un rabâchage de règles aussi antiques qu'absurdes à propos d'œuvres sclérosées. Lucie Bourassa démontre le contraire dans sa remarquable étude publiée aux éditions Balzac, Rythme et Sens ${ }^{1}$. D'une part, en oubliant le système traditionnel de description du vers régulier français pour fonder son travail sur une définition plus vaste du rythme. D'autre part, en appliquant son analyse à des œuvres contemporaines.

Dans la première partie de son ouvrage, Lucie Bourassa s'attache à établir une définition du rythme suffisamment englobante pour justifier l'importance qu'elle attribue au rythme dans la signification même de l'œuvre littéraire. Dans ce but, elle commence par faire le point sur les recherches actuelles dans le domaine du rythme. Cette démarche donne au volume une allure de thèse un peu rébarbative. Émile Benveniste, Henri Meschonnic, Jacques Garelli, entre autres, sont appelés à la barre; puis Jean-Claude Milner et François Régnault. Cependant, ce résumé des critiques les dépasse aisément: par la minutie et l'intelligence de ses analyses, en effet, par son implication dynamique dans la critique des diverses théories, $\mathrm{M}^{\mathrm{me}}$ Bourassa offre d'abord un panorama ordonné de la question puis elle réussit à extraire de ces théories diverses un certain nombre de définitions précises et d'instruments d'analyse opératoires. La valeur première de Rythme et Sens tient, je crois, à la qualité de cette synthèse, des plus éclairantes, d'un champ de recherche passablement complexe.

Une ouvre littéraire est à la fois comprise et perçue, devinée et ressentie. "Lire, écrire un poème c'est éprouver le sens autant que le comprendre. Tout se passe comme si la poésie mettait en œuvre non seulement du sens, mais aussi, et simultanément, du sens "sensible" (p. 403). La lecture se déroule dans une certaine durée et retrouve la durée de la création. Cette durée est inscrite dans la structure de l'œuvre: ainsi le rythme, loin de se limiter à un agencement gratuit de syllabes, mais "envisagé comme organisation particulière d'une temporalité discursive" (p. 13), est la vie même de l'œuvre, présente à tous les niveaux, depuis les cellules que constituent les syllabes, en passant 
par les phrases, les strophes, jusqu'au squelette des grandes parties ou des chapitres. Créé par l'écriture, recréé par la lecture, le rythme est la manière dont le sens se réalise dans la durée d'un discours. Conception du rythme et conception du temps sont donc liées. Et $\mathrm{M}^{\mathrm{me}}$ Bourassa fait un excellent rappel (autant que je puisse juger) des considérations philosophiques qui constituent l'arrière-plan des conceptions du rythme littéraire: temps vécu et mémoire, perception des alternances régulières dans la nature (rythme diurne, rythme de la mer, rythme cardiaque ou respiratoire, etc.). Tout rythme, naturel ou artistique, temporel ou autre, peut être considéré comme une "manière particulière de fluer" (Benveniste) (p. 33), une succession (assez régulière pour être perçue) de "contrastes" et de "retours" (p. 65).

Dans les productions du langage, $\mathrm{M}^{\mathrm{me}}$ Bourassa reprend la distinction de Meschonnic entre "rythme linguistique" (inscrit dans le code de chaque langue) et "rythme poétique" défini comme l'"organisation d'une écriture", soit l'" organisation subjective des marques" (p. 68). Tout discours, littéraire ou non littéraire, est donc rythmé, chacun à sa manière. La particularité du texte poétique est de donner une plus grande importance au rythme, à la fois en le resserrant, en le régularisant (dans la métrique traditionnelle), et en en faisant un médium de signification essentiel.

La deuxième partie de Rythme et Sens vise à élaborer une méthode précise d'analyse du rythme dans des ouvres poétiques ne se pliant pas aux règles de la métrique traditionnelle. De prime abord, je n'ai pu m'empêcher d'être surpris du décalage entre l'ampleur, la complexité et la profondeur des réflexions sur le rythme dans sa généralité, qui constituent la première partie, et le retour aux questions de compte de syllabes, de distinction des divers types d'accents linguistiques et autres byzantinismes de cette deuxième partie. La première laissait espérer une méthode moins terre-à-terre, plus enlevante. Sans doute est-ce le prix d'une démarche scientifique. Mais l'articulation entre la première partie, plus philosophique et anthropologique, et la deuxième, plus linguistique, ne m'a pas paru évidente.

Cependant, toujours avec la même minutie et la même intelligence, $\mathrm{M}^{\text {me }}$ Bourassa y reprend les distinctions habituelles dans ce type d'ouvrages, sur les accents toniques et les accents initiaux, les groupes rythmiques ("mots phonologiques"), les mètres et les vers. Reconnaissant la dimension subjective de la détermination de ces diverses marques, critiquant Meschonnic avec modération mais avec précision, elle en déduit une méthode d'analyse des plus intéressantes. Sa critique de l'utilisation par Meschonnic des accents initiaux dans l'analyse du rythme 
poétique est un modèle de fermeté respectueuse: "Que les consonnes initiales faisant partie d'un réseau de récurrences puissent être considérées $[. .$.$] ; que même ces séries puissent être [...] - constitue une hy-$ pothèse intéressante. Ce qui est moins clair [...]" (p. 152). Elle tire de cette réflexion critique des schémas analytiques très éclairants: avec une patience extrêmement méritoire, elle propose pour finir l'analyse rythmique d'un même texte de Michel van Schendel selon la méthode de Meschonnic, celle de Milner et Régnault, et la sienne (p. 168-171). $\mathrm{Au}$ lecteur de constater comment elle a réussi à simplifier et à rendre ainsi plus efficaces les méthodes de ses prédécesseurs.

Toutefois, je m'interroge sur la pertinence de telles analyses sur des poèmes modernes en vers libres. Que ce type d'analyse, reconstituant syllabe par syllabe le rythme précis de chaque vers, soit des plus utiles dans le cas d'œuvres particulières ou de vers particuliers, je n'en discute pas. Mais qu'elle constitue la base systématique d'une étude de la poésie contemporaine peut apparaître comme une généralisation excessive. Étant donné que la grande majorité des vers actuels ne sont pas "comptés" (le poète ne compte pas les syllabes pour créer des rythmes spécifiques), ce niveau d'analyse est peut-être d'un pointillisme exagéré. Et il faudrait se demander si ce qui se justifiait dans le cas du vers régulier (puisqu'il était toujours identique en longueur, l'analyse du rythme devait donc se situer au niveau infra-métrique, dans le détail de la structure interne du mètre), se justifie encore face au vers libre qui, naturellement ou par définition, est fondé sur la diversité, sur l'irrégularité.

L'analyse ne serait-elle pas plus concluante si elle se plaçait d'abord à un niveau plus large? C'est précisément ce que propose la suite de Rytbme et Sens, passant du démontage des "mots phonologiques " à la comparaison des "groupes supérieurs ", éléments de phrases délimités par des signes de ponctuation ou des blancs. Le chapitre "Intonation et groupes supérieurs " étudie le mouvement des phrases, l'enchaînement des segments ou des vers en une mélodie du texte. Il me semble que cette direction de l'analyse du rythme est très prometteuse et qu'elle mériterait d'être développée au niveau des groupes encore plus "supérieurs": la strophe, la séquence, le poème par rapport au recueil. Là se situe, aussi, le rythme de l'œuvre. Le dernier chapitre de cette seconde partie se penche enfin sur la question des sonorités dans leur relation avec le rythme. Traditionnellement, le jeu des phonèmes, allitérations, assonances, rimes, constituait un champ nommé "harmonie". Je viens d'employer le terme de "mélodie" et je me demande si, en relation avec les remarques précédentes sur les "groupes supérieurs", relier l'analyse des phonèmes à celle d'une 
"mélodie du texte" ne serait pas plus pertinent que de les limiter à être une marque rythmique parmi d'autres.

La troisième partie de l'ouvrage de Lucie Bourassa propose l'étude de poèmes de trois auteurs contemporains: Jean Tortel, André du Bouchet et Michel van Schendel. Il s'agit de textes de formes diverses, en prose ou en vers, avec ou sans effets de spatialisation dans la page. Les analyses de $\mathrm{M}^{\mathrm{me}}$ Bourassa sont systématiques et rigoureuses. Ses déductions se limitent volontairement à ce qui peut être établi solidement. Ses conclusions peuvent ainsi paraître limitées, elles sont objectives. Dans l'œuvre de Michel van Schendel, elle s'arrête d'abord aux réflexions théoriques sur la poésie et le rythme de l'auteur de $L^{\prime} E x$ trême Livre des voyages. Le poète qui a écrit que "le rythme fait le sens et n'oublie pas la référence" (p. 339), ne pouvait qu'intéresser notre critique. De sa lecture des poèmes de van Schendel, elle retire:

Le rythme assume un rôle actif au cour de la dynamique de tensions et de paradoxes qui anime non seulement la poétique, mais aussi la poésie de cet écrivain. C'est le rapport entre le rythme comme *inscription actuelle , , événement circonstancié et singulier du langage - et comme inscription "historique" du "sens" à "intensité diachronique variable", qui fonde la cohérence de l'œuvre (p. 395).

Dans sa conclusion, Lucie Bourassa reprend la même expression pour définir, d'une manière plus générale, le rythme poétique comme une "dynamique de tensions" (p. 406), avant de distinguer divers types de tensions. Continuité et discontinuité, sous les différentes formes qu'elles prennent dans le texte poétique, nous renvoient en fin de compte à la dialectique éternelle du même et de l'autre.

1. Lucie Bourassa, Rythme et Sens. Des processus rythmiques en poésie contemporaine, Montréal, Balzac, 1993, 455 p. 